\title{
Isomers and shell evolution in neutron-rich nuclei below the doubly magic nucleus ${ }^{132} \mathrm{Sn}$
}

\author{
Hiroshi Watanabe $\mathrm{e}^{1,2, *}$ \\ ${ }^{1}$ IRCNPC, School of Physics and Nuclear Energy Engineering, Beihang University, Beijing 100191 China \\ ${ }^{2}$ RIKEN Nishina Center, 2-1 Hirosawa, Wako, Saitama 351-0198, Japan
}

\begin{abstract}
The level structures of the very neutron-rich nuclei ${ }^{128} \mathrm{Pd}_{82}$ and ${ }^{126} \mathrm{Pd}_{80}$ have been investigated for the first time. A new isomer with a half-life of $5.8(8) \mu \mathrm{s}$ in ${ }^{128} \mathrm{Pd}$ is proposed to have a spin and parity of $8^{+}$ and is associated with a maximally aligned configuration arising from the $g_{9 / 2}$ proton subshell with seniority $v=2$. The level sequence below the $8^{+}$isomer is similar to that in the $N=82$ isotone ${ }^{130} \mathrm{Cd}$, but the electric quadrupole transition that depopulates the $8^{+}$isomer is more hindered in ${ }^{128} \mathrm{Pd}$ than in ${ }^{130} \mathrm{Cd}$, as expected in the seniority scheme for a semi-magic, spherical nucleus. For ${ }^{126} \mathrm{Pd}$, three new isomers with $J^{\pi}=\left(5^{-}\right),\left(7^{-}\right)$, and $\left(10^{+}\right)$have been identified with half-lives of 0.33(4) $\mu \mathrm{s}, 0.44(3) \mu \mathrm{s}$, and 23.0(8) $\mathrm{ms}$, respectively. The smaller energy difference between the $10^{+}$and $7^{-}$isomers in ${ }^{126} \mathrm{Pd}$ than in the heavier $N=80$ isotones can be interpreted as being ascribed to the monopole shift of the $h_{11 / 2}$ neutron orbit. The nature of the $N=82$ shell closure scrutinized with these characteristic isomers is discussed.
\end{abstract}

\section{Introduction}

The concept of magicity is of supreme importance for many-body fermionic systems in a confined space. The stability of atomic nuclei, which consist of a number of sub-atomic particles called protons and neutrons (nucleons), is much influenced by a shell structure and its resulting magic numbers; nuclei with specific numbers of nucleons $(2,8,20,28,50,82$ both for protons and for neutrons, and also 126 for neutrons) near the $\beta$-stability line necessitate relatively high energies to remove one or two nucleons compared to the neighboring isotopes. For the last few decades, however, the study of exotic nuclei using radioactive isotope (RI) beams revealed that the aforementioned magic numbers are not necessarily universal and are subjected to a change in some regions of light-mass nuclei with highly unbalanced ratios of protons and neutrons $[1,2]$. Such a paradigm shift is one of the frontier issues in nuclear physics, and whether it will also take place at the heavier traditional magic numbers is an open question worth investigating.

The present work focuses on neutron-rich nuclei below the doubly magic nucleus ${ }^{132} \mathrm{Sn}$. It is still under debate that the $N=82$ spherical shell gap is quenched as approaching the neutron drip line. From an astrophysical point of view, it has been argued that the property of the $N=82$ shell closure far from the valley of $\beta$-stability affects the Solar System abundance pattern particularly around the prominent $A \approx 130$ peak in the $r$-process nucleosynthesis [3], and hence, a proper understanding of the underlying nuclear structure of the $r$-process isotopes is highly required. The characteristic isomers that involve high- $j$ orbits, such

*e-mail: hiroshi@ribf.riken.jp as $\pi g_{9 / 2}$ and $v h_{11 / 2}$, in their configurations can serve as a sensitive probe for the evolution of shell structures in this exotic region. We have performed decay spectroscopy experiments at the RI Beam Factory (RIBF) facility [4], which has the capability of providing the world's strongest RI beams, as part of the EURICA campaign using highintensity ${ }^{238} \mathrm{U}$ beams. New results obtained include the seniority isomer with $J^{\pi}=\left(8^{+}\right)$in ${ }^{128} \mathrm{Pd}$ [5], the longlived $\left(10^{+}\right)$isomeric state in ${ }^{126} \mathrm{Pd}[6]$, and a high-spin $\beta$ decaying isomer in ${ }^{127} \mathrm{Ag}$ [7]. In this report, the nature of the $N=82$ shell closure will be discussed in terms of the seniority isomerism in the $N=82$ isotones, as well as the effect of the monopole interaction between the $g_{9 / 2}$ proton and $h_{11 / 2}$ neutron subshells.

\section{Experimental procedures}

Neutron-rich isotopes below the doubly magic nucleus ${ }^{132} \mathrm{Sn}$ have been produced using in-flight fission of a ${ }^{238} \mathrm{U}^{86+}$ beam accelerated up to $345 \mathrm{MeV} / \mathrm{u}$ by a series of accelerators at RIBF facility [4], cooperated by RIKEN Nishina Center and CNS, University of Tokyo. The primary beam with the intensity ranging from 7 to $12 \mathrm{pnA}$ impinged on a 3-mm thick beryllium target. The nuclei of interest were separated and transported through the BigRIPS separator and the following ZeroDegree spectrometer [9], operated with wedge-shaped aluminum degraders with thicknesses of 2.9 and $2.5 \mathrm{~mm}$ at the first and second dispersive focal planes, respectively, for purification of the secondary beams. Identification of particles with the atomic number $(Z)$ and the mass-to-charge ratio $(A / Q)$ was achieved on the basis of the $\Delta E$-TOF- $B \rho$ method, in which the energy loss $(\Delta E)$, time of flight (TOF), and mag- 


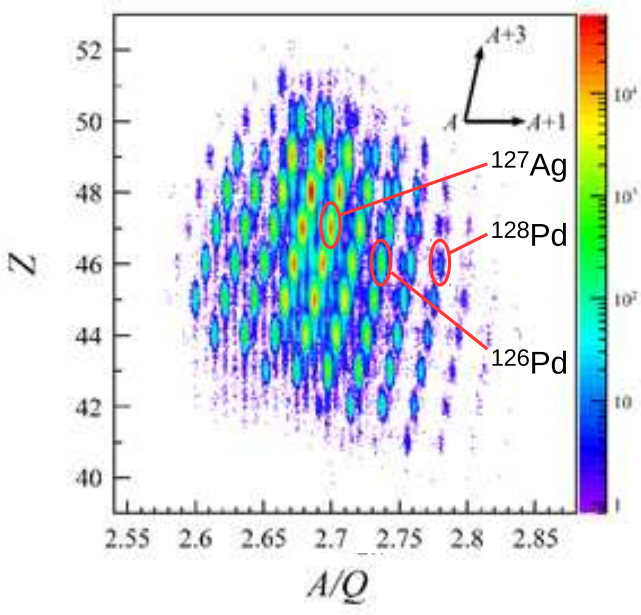

Figure 1. Particle-identification spectrum obtained in the present work. ${ }^{127} \mathrm{Ag}^{47+}$ and ${ }^{126,128} \mathrm{Pd}^{46+}$ ions are indicated with circles. The figure is taken from Ref. [8].

netic rigidity $(B \rho)$ were measured using the focal-plane detectors on the beam line [10]. A particle-identification spectrum obtained in the present experiment is displayed in Fig. 1.

The identified fragments were implanted into a highly segmented active stopper, named WAS3ABi [11], which consisted of eight double-sided silicon-strip detectors (DSSSD) stacked compactly. Each DSSSD had a thickness of $1 \mathrm{~mm}$ with an active area segmented into sixty and forty strips (1-mm pitch) on each side in the horizontal and vertical dimensions, respectively. The DSSSDs also served as detectors for electrons following $\beta$-decay and internal conversion (IC) processes. Gamma rays were detected by the EURICA array [12] that consisted of twelve Cluster-type detectors, each of which contained seven HPGe crystals packed closely. The $\gamma$-ray measurements were carried out with a time condition up to $100 \mu \mathrm{s}$ relative to the trigger signal generated either from a plastic scintillation counter placed at the end of the beam line or from WAS3ABi.

The beam, electron, and $\gamma$-ray events were timestamped and recorded by independent data-acquisition systems. For the analysis of beam- $\gamma$ (delayed) coincidence, the $\gamma$-ray data sets were combined with those of the beam particles on an event-by-event basis using information on the time stamp. Isomeric states with (sub)microsecond lifetimes were identified with appropriate time gates. All data sets containing beam, electron, and $\gamma$-ray events were used for $\beta-\gamma$ and IC- $\gamma$ coincidence analyses, in which the implantation of an identified particle was associated with the subsequent electron events that were detected in the same DSSSD pixel. Decay halflives $\left(T_{1 / 2}\right)$ in the millisecond range were extracted from the time distributions of $\gamma$-ray gated electron events with respect to the fragment implantation.

\section{Results and discussion}

\section{$3.1{ }^{128} \mathbf{P d}_{82}$}

Figure 2(a) shows a $\gamma$-ray energy spectrum measured in delayed coincidence with ${ }^{128} \mathrm{Pd}$ ions. Four $\gamma$ rays at energies of 75, 260, 504, and $1311 \mathrm{keV}$ have been unambiguously observed. These $\gamma$ rays are found to be in mutual coincidence and exhibit consistent time behavior. Therefore, we conclude that they proceed through a single cascade originating from one isomeric state. A least-squares fit of the summed gated time spectra of the isomeric-decay transitions yields $T_{1 / 2}=5.8(8) \mu \mathrm{s}$. The total internal conversion coefficient $\left(\alpha_{T}\right)$ for the $75-\mathrm{keV}$ transition derived from a comparison with the $1311-\mathrm{keV} \gamma$-ray intensity is $2.6(17)$, which is consistent with the theoretical value of $\alpha_{T}=3.88$ for an E2 multipolarity. Based on these arguments, the level scheme of ${ }^{128} \mathrm{Pd}$ is proposed as displayed in Fig. 3, where the spin and parity of the $5.8-\mu$ s isomeric state at $2151 \mathrm{keV}$ is assigned as $J^{\pi}=8^{+}$. A transition strength of $B\left(E 2 ; 8^{+} \rightarrow 6^{+}\right)=0.22(3)$ W.u. can be obtained from the measured half-life of the $2151-\mathrm{keV}$ isomeric state.

Assuming that the excited states in ${ }^{128} \mathrm{Pd}$ are the same multiplet members (with seniority $v=2$, as will be discussed later), the half-life of the $J^{\pi}=6^{+}$state is estimated to be $22(3) \mathrm{ns}$ from that of the $8^{+}$isomer. Since this value is significantly shorter than the flight time of fragments from the production target to the implantation position ( $~$ $650 \mathrm{~ns}$ ), there would be little ${ }^{128} \mathrm{Pd}$ ions remaining at this state during the flight. Hence, the observed intensities of the four cascade $\gamma$ rays should depend only on the population of the $8^{+}$isomeric state.

The excitation energies of the $J^{\pi}=2^{+}-8^{+}$states in ${ }^{128} \mathrm{Pd}$ are comparable to those in ${ }^{130} \mathrm{Cd}[13]$. The constancy of level energies is characteristic of the seniority scheme, where seniority $v$ counts the number of nucleons that are not in pairs coupled to spin zero. In case of a $n$ particle (or $n$-hole) system in a single- $j$ shell, the level energies with identical $J^{\pi}$ and $v$ are independent of $n$. Thus, the excited states in ${ }^{128} \mathrm{Pd}$ can be interpreted in terms of the $v=2$ configuration of the $\pi g_{9 / 2}$ subshell, which locates just below the $Z=50$ shell gap.

A direct implication of the $N=82$ shell closure can be corroborated from the behavior of the $E 2$ transition strength for the $8^{+} \rightarrow 6^{+}$isomer decay in ${ }^{128} \mathrm{Pd}$. The $E 2$ matrix elements between $v=2$ states are close to zero near the middle of the valence shell in semi-magic nuclei, giving rise to seniority isomerism. For the $N=82$ isotones, it turns out that the $B\left(E 2 ; 8^{+} \rightarrow 6^{+}\right)$value is much smaller in ${ }^{128} \mathrm{Pd}$ than in ${ }^{130} \mathrm{Cd}$, as expected in the exact seniority classification, namely, $B(E 2 ; v=2, n=4)=\frac{1}{9} B(E 2 ; v=$ $2, n=2)$. This observation indicates that both the $J^{\pi}=8^{+}$ and $6^{+}$states in the $N=82$ isotones have good seniority $v=2$ in the well isolated $\pi g_{9 / 2}$ subshell, in consequence of the robust shell closure.

\section{$3.2{ }^{126} \mathbf{P d}_{80}$}

The level scheme of ${ }^{126} \mathrm{Pd}$ constructed in the current work is also shown in Fig. 3. The 693-keV $\gamma$ ray, which is 

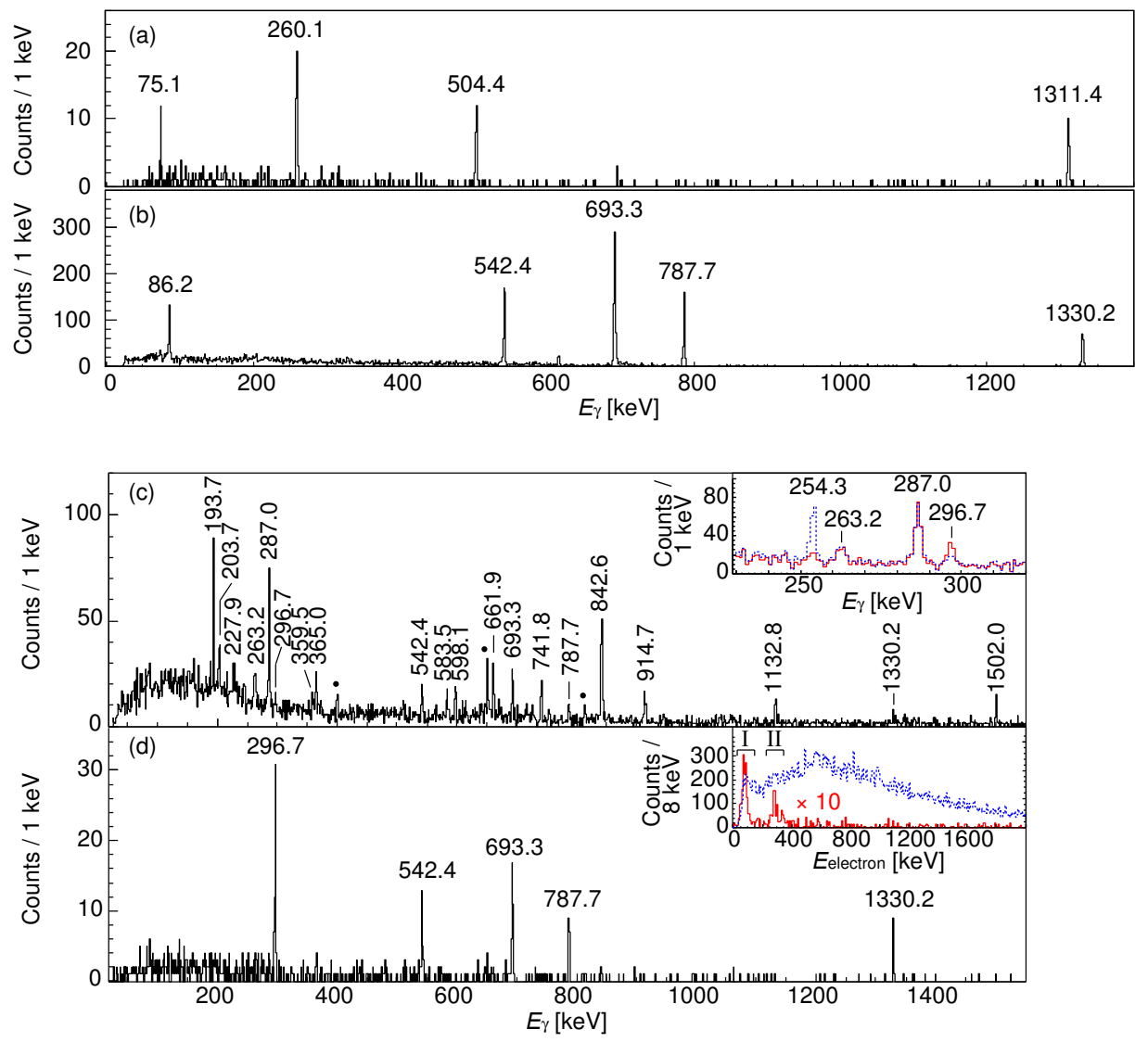

Figure 2. Gamma-ray spectra measured (a) in coincidence with ${ }^{128} \mathrm{Pd}$ ions within a time range of $0.15-25 \mu \mathrm{s}$, (b) in coincidence with ${ }^{126} \mathrm{Pd}$ ions within $0.15-5 \mu \mathrm{s}$, and (c) within $50 \mathrm{~ms}$ after the ${ }^{126} \mathrm{Pd}$ implantation with a gate on an electron- $\gamma$ time difference of $-0.5 \leq \Delta t_{e \gamma} \leq 0.5 \mu \mathrm{s}$. Contaminants from the granddaughter ${ }^{126} \mathrm{Cd}$ are marked with filled circles. The inset magnifies the energy region from 230 to $310 \mathrm{keV}$ measured with $-4.0 \leq \Delta t_{e \gamma} \leq 0.5 \mu \mathrm{s}$ (red) and $-0.5 \leq \Delta t_{e \gamma} \leq 50 \mu \mathrm{s}$ (blue). (d): $\gamma$ rays measured with $-4.0 \leq \Delta t_{e \gamma} \leq 0.5 \mu \mathrm{s}$, and additionally, with a gate on the electron peak marked with "I" in the inset, where the spectrum depicted with the red line (multiplied by a factor of ten) is obtained with a sum of gates on the $\gamma$ rays below the (5-) isomer in ${ }^{126} \mathrm{Pd}$, while the blue line represents an electron spectrum without $\gamma$-ray gates.

the most intense peak of those observed in delayed coincidence with ${ }^{126} \mathrm{Pd}$ ions [Fig. 2(b)], is assigned as the $2^{+} \rightarrow 0^{+}$transition. The coincidence spectrum with a gate on the 542-keV $\gamma$ ray clearly exhibited $\gamma$ rays at energies of 693,788 , and $86 \mathrm{keV}$, but not at $1330 \mathrm{keV}$, indicating that two parallel cascades stem from a common level at 2023 $\mathrm{keV}$. The $86-\mathrm{keV}$ transition is placed just above the 2023$\mathrm{keV}$ level, because this $\gamma$ ray was observed to precede all the four transitions.

A half-life of $0.44(3) \mu$ s is derived from the time distribution of the 86-keV $\gamma$ ray relative to the beam implantation, while the time difference between the $86-\mathrm{keV} \gamma$ ray and the subsequent transitions provides $T_{1 / 2}=0.33$ (4) $\mu \mathrm{s}$. Thus, it is found that two isomeric states lie at 2023 and $2110 \mathrm{keV}$. The assignment of spins and parities for the two isomeric states is based on the $\gamma$-ray feeding pat- tern and transition probabilities. Given the $J^{\pi}=5^{-}$ and $7^{-}$assignments for the 2023- and 2110-keV levels, respectively, the strengths of the isomeric decay transitions are $B\left(E 1 ; 5^{-} \rightarrow 4^{+}\right)=2.9(4) \times 10^{-9}$ W.u. $(542$ $\mathrm{keV}), B\left(E 3 ; 5^{-} \rightarrow 2^{+}\right)=0.24(3)$ W.u. $(1330 \mathrm{keV})$, and $B\left(E 2 ; 7^{-} \rightarrow 5^{-}\right)=2.13(14)$ W.u. $(86 \mathrm{keV})$. These strengths are comparable with the values of the corresponding transitions in the $N=80$ isotone ${ }^{128} \mathrm{Cd}$ [14].

The $\gamma$ rays below these isomers, except for the $86-\mathrm{keV}$ line, have been also observed in coincidence with electrons that were associated with the prior implantation of ${ }^{126} \mathrm{Pd}$, as demonstrated in Fig. 2(c). This observation implies the existence of a long-lived, higher-spin isomer which decays via the cascades that include electromagnetic transitions with relatively large total conversion coefficients. With gates on these $\gamma$ rays, a prominent peak can be found 

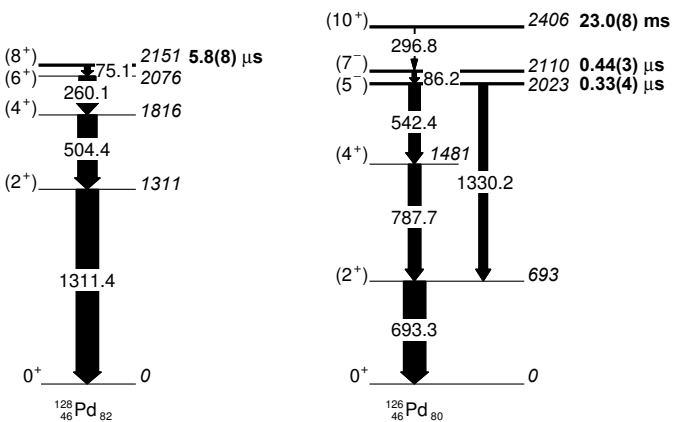

Figure 3. Partial level schemes of ${ }^{128} \mathrm{Pd}$ (left) and ${ }^{126} \mathrm{Pd}$ (right) established in the present work [5, 6].

in an electron spectrum [marked with "I" in the inset of Fig. 2(c)]; this corresponds to the conversion electrons for the 86-keV, E2 transition ( $\left.\alpha_{T}=2.374\right)$. In Fig. 2(d), a $\gamma$ ray at $297 \mathrm{keV}$ is clearly visible in addition to the $\gamma$ rays below the $\left(5^{-}\right)$isomer by gating on the $86-\mathrm{keV}$ IC peak. The appearance of the $297-\mathrm{keV}$ peak is emphasized by taking a $\gamma$-ray time condition earlier than electron events, as is evident from the inset of Fig. 2(c), suggesting that this new $\gamma$ ray precedes the highly converted $86-\mathrm{keV}$ transition. Thus, the long-lived isomer can be identified at an excitation energy of $2406 \mathrm{keV}$. A peak marked with "II" in the inset of Fig. 2(d) is expected to arise from the conversion electrons for the 297-keV transition, being most likely of an $E 3$ character $\left(\alpha_{T}=0.1197\right)$. In addition to the internaldecay branch, $\beta$ decay from the long-lived isomer was observed to populate excited states at high spins in ${ }^{126} \mathrm{Ag}$. The isomeric half-life was determined to be $23.0(8) \mathrm{ms}$ by taking a weighted average of those derived from the time distributions of the internal- and external-decay branches.

The main surprise in the present work is the small energy difference between the $\left(10^{+}\right)$and $\left(7^{-}\right)$isomers, $\Delta E_{7^{-}}^{10^{+}}$, in ${ }^{126} \mathrm{Pd}$, compared to the analogous levels in the heavier $N=80$ isotones, as demonstrated in Fig. 4 . Since these two levels consist predominantly of maximally aligned two neutron-hole configurations, $\left(v 1 h_{11 / 2}^{-2}\right)_{10^{+}}$and $\left(v 1 h_{11 / 2}^{-1} 2 d_{3 / 2}^{-1}\right)_{7^{-}}$, their level energies depend on the singleparticle energies (SPEs) of the $v 1 h_{11 / 2}$ and $v 2 d_{3 / 2}$ neutron orbits, as well as the strength of interactions between them. It can be seen in Fig. 4 that the energy of the $11 / 2^{-}$level relative to the $3 / 2^{+}$ground state in the neighboring $N=81$ isotones decreases when approaching $Z=50$. Note that these states are of one neutron-hole nature, and that the smooth reduction in energy can be essentially interpreted in terms of a short-range proton-neutron interaction as follows: The $\pi 1 g_{7 / 2}$ proton orbit lies just above the $Z=50$ shell closure. The monopole interaction between $\pi 1 g_{7 / 2}$ and $v 1 h_{11 / 2}$, a spin-flip pair with $\Delta \ell=1$ and $\Delta n=0$, is stronger than the $\pi 1 g_{7 / 2}-v 2 d_{3 / 2}$ pair, due to the larger overlap between the radial wave functions of the two orbits. Therefore, when emptying the $\pi 1 g_{7 / 2}$ subshell, the

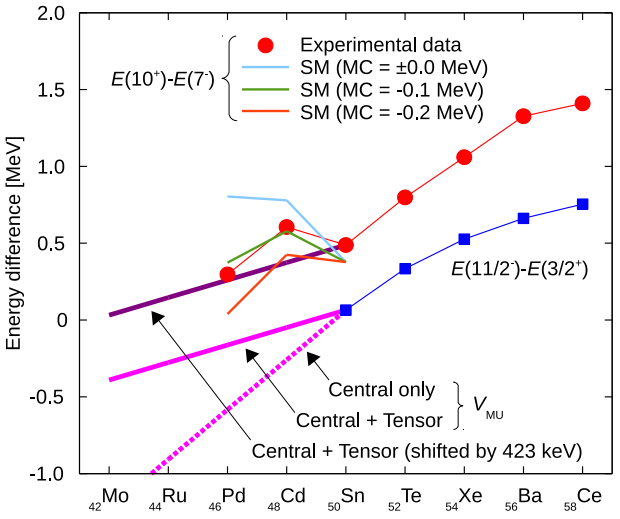

Figure 4. Energy differences between the $10^{+}$and $7^{-}$states, $\Delta E_{7^{-}}^{10^{+}}$, in $N=80$ (filled circles) and between the $11 / 2^{-}$and $3 / 2^{+}$ states in $N=81$ (filled squares). For $Z \leq 50$, the differences in energy between the $1 h_{11 / 2}$ and $2 d_{3 / 2}$ neutron orbits calculated by $V_{\mathrm{MU}}$ interaction [15] and $\Delta E_{7^{-}}^{10^{+}}$by shell-model (SM) calculations with monopole corrections (MC) are also depicted.

$v 1 h_{11 / 2}$ orbit is relatively less bound than the $v 2 d_{3 / 2}$ one. The moderation in the slope at higher $Z$ may be ascribed to the partial occupation of the $\pi 2 d_{5 / 2}$ orbit, which exerts a weaker (stronger) monopole interaction on the $v 1 h_{11 / 2}$ $\left(v 2 d_{3 / 2}\right)$ neutrons than $\pi 1 g_{7 / 2}$. As is evident from the comparison in Fig. 4, a similar trend is observed for $\Delta E_{7^{-}}^{10^{+}}$. This finding supports that these isomers can serve as sensitive probes for the evolution of the constituent neutron shell orbits.

Below $Z=50$, protons in the $\pi 1 g_{9 / 2}$ subshell play a major role in changing the neutron SPEs, because this orbit is near the Fermi surface. The neutron SPEs are estimated by the monopole-based universal interaction, $V_{\mathrm{MU}}$, which consists of the Gaussian central force and the tensor force based on the $\pi+\rho$ meson exchanges, using the parameters fixed in Ref. [15]. The $V_{\mathrm{MU}}$ interaction has been applied to other regions of exotic nuclei and successfully reproduced various types of the shell evolution [15]. Figure 4 shows the evolutions of the relative $11 / 2^{-}$energy starting from the experimental value in ${ }^{131} \mathrm{Sn}$, predicted by the $V_{\mathrm{MU}}$ calculation with $A=130$. In the calculation, the $\pi 1 g_{9 / 2}$ subshell is considered to be well isolated between $Z=50$ and 40. In Fig. 4, only with the central force the $11 / 2^{-}$energy rapidly decreases to negative values as the proton number decreases, indicating that the $v 1 h_{11 / 2}$ orbit moves away from the $v 2 d_{3 / 2}$ one towards the $N=82$ gap. If there is the tensor force effect, however, the slope becomes less steep. The observed reduction in $\Delta E_{7^{-}}^{10^{+}}$from ${ }^{130} \mathrm{Sn}$ to ${ }^{126} \mathrm{Pd}$ is consistent with the expectation of $V_{\mathrm{MU}}$ including both the central and tensor forces, given the same trend as the $11 / 2^{-}$relative energies. Based on these findings, it can be concluded that the tensor force slows down the upward drift of the $v 1 h_{11 / 2}$ SPE when protons are removed from the $\pi 1 g_{9 / 2}$ orbit below ${ }^{132} \mathrm{Sn}$. 
One can notice a kink in the systematics of $\Delta E_{7^{-}}^{10^{+}}$at ${ }^{128} \mathrm{Cd}$ in Fig. 4. While the overall trend can be accounted for by $V_{\mathrm{MU}}$, other multipole components have to be introduced in a shell-model framework to describe the details of the level evolution. To confirm the effect of configuration mixing, a shell-model (SM) study based on the $j-j$ coupling scheme was performed. With the doubly magic nucleus ${ }^{132} \mathrm{Sn}$ as a closed core, the model space adopted includes the $2 d_{3 / 2}, 1 h_{11 / 2}, 3 s_{1 / 2}, 2 d_{5 / 2}$, and $1 g_{7 / 2}$ orbits for neutron holes, and the $1 g_{9 / 2}, 2 p_{1 / 2}, 2 p_{3 / 2}, 1 f_{5 / 2}$ orbits for proton holes. The SPEs were taken from the experimentally known levels in the single-hole nuclei, ${ }^{131} \mathrm{Sn}$ and ${ }^{131} \mathrm{In}$. The surface-delta interaction was employed for the proton-proton and neutron-neutron interactions, which were adjusted to reproduce the observed level energies of ${ }^{130} \mathrm{Cd}$ and ${ }^{130} \mathrm{Sn}$, respectively. The proton-neutron two-body matrix elements (TBME) were derived from the empirical parameterization of interaction potentials [16]. For the $\pi 1 g_{9 / 2}-v 1 h_{11 / 2}$ multiplet, the monopole correction (MC) was introduced by adding a constant to the diagonal TBME. The same idea of MC was adopted in largescale shell-model calculations for neutron-rich nuclei in the south-west quadrant of ${ }^{132} \mathrm{Sn}$ [17].

The results of SM calculations with different values of the monopole correction for $Z \leq 50$ are displayed in Fig. 4. The kink manifests in the calculated $\Delta E_{7^{-}}^{10^{+}}$, as well as in the experimental result. Moreover, it can be seen that the variation in $\Delta E_{7^{-}}^{10^{+}}$is very sensitive to the value of the monopole correction. According to the present SM calculation, the wave functions of the $10^{+}$and $7^{-}$states in each $N=80$ isotone are scarcely affected by the monopole corrections. These arguments indicate that the lowering of $\Delta E_{7^{-}}^{10^{+}}$in ${ }^{126} \mathrm{Pd}$ is ascribed to the monopole effect between the $\pi 1 g_{9 / 2}$ and $v 1 h_{11 / 2}$ orbitals rather than the configuration mixing of any kind.

\section{Summary}

This paper reports on the experimental results of characteristic isomers in ${ }^{126,128} \mathrm{Pd}$ studied as part of the EURICA experimental campaign in 2012 at RIBF. The data analysis and shell-model calculations for ${ }^{127} \mathrm{Ag}$ are still ongoing.

\section{References}

[1] O. Sorlin, M.G. Porquet, Prog. Part. Nucl. Phys. 61, 602 (2008)
[2] T. Nakamura, H. Sakurai, H. Watanabe, Prog. Part. Nucl. Phys. 97, 53 (2017)

[3] K. Kratz, B. Pfeiffer, O. Arndt, Eur. Phys. J. A 25, 633 (2005)

[4] Y. Yano, Nucl. Instrum. Methods Phys. Res. B 261, 1009 (2007)

[5] H. Watanabe, G. Lorusso, S. Nishimura, Z.Y. Xu, T. Sumikama, P.A. Söderström, P. Doornenbal, F. Browne, G. Gey, H.S. Jung et al., Phys. Rev. Lett. 111, 152501 (2013)

[6] H. Watanabe, G. Lorusso, S. Nishimura, T. Otsuka, K. Ogawa, Z.Y. Xu, T. Sumikama, P.A. Söderström, P. Doornenbal, Z. Li et al., Phys. Rev. Lett. 113, 042502 (2014)

[7] H. Watanabe, (to be submitted)

[8] Y. Shimizu, T. Kubo, N. Fukuda, N. Inabe, D. Kameda, H. Sato, H. Suzuki, H. Takeda, K. Yoshida, G. Lorusso et al., Journal of the Physical Society of Japan 87, 014203 (2018)

[9] T. Kubo, D. Kameda, H. Suzuki, N. Fukuda, H. Takeda, Y. Yanagisawa, M. Ohtake, K. Kusaka, K. Yoshida, N. Inabe et al., Progress of Theoretical and Experimental Physics 2012, 03 C003 (2012)

[10] N. Fukuda, T. Kubo, T. Ohnishi, N. Inabe, H. Takeda, D. Kameda, H. Suzuki, Nucl. Instrum. Methods B 317, 323 (2013)

[11] S. Nishimura, Progress of Theoretical and Experimental Physics 2012, 03 C006 (2012)

[12] P.A. Söderström, S. Nishimura, P. Doornenbal, G. Lorusso, T. Sumikama, H. Watanabe, Z. Xu, H. Baba, F. Browne, S. Go et al., Nucl. Instrm. Methods B 317, 649 (2013)

[13] A. Jungclaus, L. Cáceres, M. Górska, M. Pfützner, S. Pietri, E. Werner-Malento, H. Grawe, K. Langanke, G. Martínez-Pinedo, F. Nowacki et al., Phys. Rev. Lett. 99, 132501 (2007)

[14] L. Cáceres, M. Górska, A. Jungclaus, M. Pfützner, H. Grawe, F. Nowacki, K. Sieja, S. Pietri, D. Rudolph, Z. Podolyák et al., Phys. Rev. C 79, 011301 (2009)

[15] T. Otsuka, T. Suzuki, M. Honma, Y. Utsuno, N. Tsunoda, K. Tsukiyama, M. Hjorth-Jensen, Phys. Rev. Lett. 104, 012501 (2010)

[16] J.P. Schiffer, W.W. True, Rev. Mod. Phys. 48, 191 (1976)

[17] H.K. Wang, K. Kaneko, Y. Sun, Phys. Rev. C 89, 064311 (2014) 